\title{
Protein Folding and Diseases
}

\author{
Cheolju Lee ${ }^{\dagger * *}$ and Myeong-Hee Yu ${ }^{\ddagger}$ \\ Life Sciences Division and "Functional Proteomics Center, Korea Institute of Science and Technology, \\ Hawolgok-dong, Seongbuk-gu, Seoul 136-791 Korea
}

Received 9 May 2005

For most of proteins to be active, they need well-defined three-dimensional structures alone or in complex. Folding is a process through which newly synthesized proteins get to the native state. Protein folding inside cells is assisted by various chaperones and folding factors, and misfolded proteins are eliminated by the ubiquitin-proteasome degradation system to ensure high fidelity of protein expression. Under certain circumstances, misfolded proteins escape the degradation process, yielding to deposit of protein aggregates such as loop-sheet polymer and amyloid fibril. Diseases characterized by insoluble deposits of proteins have been recognized for long time and are grouped as conformational diseases. Study of protein folding mechanism is required for better understanding of the molecular pathway of such conformational diseases.

Keywords: Amyloid fibril, Chaperone, Conformational disease, Protein folding, Protein misfolding

\section{Introduction}

The gene functions are manifested in the form of proteins. Proteins are central to various biological processes. In order to be biologically active, proteins must fold into their welldefined three-dimensional structures. The properties of the peptide bond and the amino acid side chains confer a high degree of conformational flexibility to the protein conformation, resulting in tremendous possible conformations from a single polypeptide chain (Fersht and Daggett, 2002). Nonetheless, only one conformation that is thermodynamically the most stable state generally corresponds to the native state, which is determined by the primary sequences. Protein folding has recently been described in terms of energy landscape of a 'folding funnel' (Dill and Chan, 1997). The bottom of the

*To whom correspondence should be addressed.

Tel: 82-2-958-6788; Fax: 82-2-958-6919

E-mail: clee270@kist.re.kr funnel represents the native state of the protein. At top of the funnel, the protein exists in random states with large number of conformations of high free energy. Progress down the funnel is accompanied by an increase in native-like structure as folding proceeds. Protein folding has been studied in detail by both experimental and theoretical procedures. In vitro folding of some simple proteins has been investigated in detail by various optical techniques including NMR, circular dichroism and fluorescence spectroscopies, with the aid of rapid-mixing devices (Fersht, 1999; Dobson, 2003). However, in vivo folding inside living cells is substantially different from in vitro folding in many aspects even though the underlying principles are the same: i) in vivo protein folding occurs in a crowded environment with a number of different proteins or different type of macromolecules; ii) protein folding sometimes occurs before completion of its synthesis.

This review describes an overview on the protein folding inside the cells. In general, folding of newly synthesized proteins is assisted by cellular chaperones; misfolded protein is monitored by quality control system and degraded by the proteasome; and aggregation of misfolded protein causes conformational diseases. Relevance of protein misfolding to some human conformational diseases will also be considered.

\section{Protein Folding in the Cell}

Many details of the folding process depend on the environment in which folding takes place. When polypeptides are synthesized in the cells, they fold in the cytoplasm after release from the ribosome or in other subcellular compartments such as endoplasmic reticula (ER) or mitochondria after they are translocated through membranes (Hartl and Hayer-Hartl, 2002; Dobson, 2004). Protein folding can also begin cotranslationally while carboxy-terminal segment of a nascent chain is inside the exit channel of ribosome (Hardesty and Kramer, 2001; Baram and Yonath, 2005). Within the cells, proteins in the process of synthesis encounter particular challenges imposed by the crowded macromolecules before completion of folding (Ellis, 2001a). As incompletely folded 
chains expose particular regions that are destined to be buried in the native state, they are prone to aggregate with other molecules because they have exposed hydrophobic surfaces. Situations are more problematic because aggregation process follows second order kinetics and therefore surpasses the firstorder folding process as their concentration increases (Fersht, 1999), which condition is normally satisfied within the concentrated milieu of the cells (Ellis, 2001a). Consequently, elaborate systems have evolved to prevent proteins from being aggregated prior to folding. The first one is molecular chaperones, and the second one is ubiquitin-proteasome system, each of which is not exclusively independent of the other, and in some way they cooperate in living cells.

Chaperones as folding assistants Chaperones are members of diverse protein families capable of binding to stabilize nonnative conformations of other proteins. They bind to the folding intermediate of polypeptides, which prevents aggregation of the intermediates and facilitates correct folding and assembly through controlled binding and release cycles (Frydman and Hartl, 1996; Ellis, 2001b; Hartl and HayerHartl, 2002). Chaperones are found in all types of cells from archaea to eukarya and various cellular compartments of the eukaryotic cell. In addition, their concentrations are increased as a response to diverse stresses such as unfolded protein response as well as the heat shock, which explains why a large number of chaperones are heat shock proteins. Overview on Hsp60s and Hsp70s, the two most studied chaperones, will provide us an insight into the mechanism of those folding assistants.

Chaperonins are a group of chaperones with molecular weight of about $60 \mathrm{kDa}$. Members include bacterial GroEL, Hsp60 of mitochondria and chloroplasts, and the TRiC in eukaryotic cytosol (Bukau and Horwich, 1998). They are characterized by the barrel-shaped double-ring structure (Braig et al., 1994). GroEL seems to be the best studied chaperonin with regard to folding mechanism (Grantcharova et al., 2001). GroEL works with GroES, a cofactor of Hsp10 family. Inside of the ring structure of GroEL, a central cavity is formed, in which an incompletely folded polypeptide is sequestered via hydrophobic interactions. The first role of GroEL is providing a protected environment to prevent the folding intermediate from sticking to one another (Ellis, 2001b). Conformational changes of the GroEL subunits driven by ATP hydrolysis induces enlargement of the cavity and a shift in its surface to a more hydrophilic lining (Barral et al., 2004). As a consequence, the polypeptide molecule can proceed on its folding pathway in the co-called 'Anfinsen cage'. GroEL also has unfolding activity. Unfolding the misfolded state will allow the protein to try further attempts at productive folding (Grantcharova et al., 2001). TRiC constitutes a different subgroup of chaperonin, because it functions independent of Hsp10 cofactor. TRiC cooperates with different upstream chaperones in the folding of distinct protein classes (Siegers et al., 2003).
Hsp70s are a well-conserved chaperone family comprising central components of the cellular network of molecular chaperones (Mayer and Bukau, 2005). They are among the first chaperones that bind newly synthesized polypeptides and are intimately involved in the translocation of unfolded polypeptides across intracellular membranes (Hartl and Hayer-Hartl, 2002). Accordingly, Hsp70 family members are found associated with ribosomes and are present in eukaryotic organelles, such as mitochondria and the ER (Munro and Pelham, 1986). In contrast to the double ring chaperonins, Hsp70 chaperones are monomeric consisting of two large globular subdomains, amino-terminal ATPase domain and a carboxy-terminal peptide binding domain (Flaherty et al., 1991; Zhu et al., 1996). Peptide-binding domain recognizes short segments of substrate polypeptide in an extended conformation (Zhu et al., 1996), which can easily be found on nascent polypeptides during translation, on the unfolded polypeptide during translocation across membranes, and on misfolded and damaged proteins (Thulasiraman et al., 1999). The ATPase cycle of Hsp70 consists of an alternation between the ATP state with low affinity and fast exchange rate for substrates, and the ADP state with high affinity and low exchange rate for substrates. Cycles of ATP binding and hydrolysis provide the basis for a dynamic interaction of Hsp70 proteins with non-native polypeptides. In a thermodynamically coupled process, peptide binding slightly stimulate the hydrolysis of ATP by Hsp70. Yet, the activity of cochaperone Hsp40 is required for further acceleration of the ATPase cycle to a physiological level (Karzai and McMacken, 1996; Barouch et al., 1997; Laufen et al., 1999). Hsp40 proteins, such as bacterial DnaJ, transiently interact with substrates and deliver them to Hsp70 in ATP-bound state (Rudiger et al., 1997). Binding of substrate and association of Hsp40 drive rapid hydrolysis of ATP leading to peptide capture and retention. Hsp70 and Hsp40 proteins apparently tightly cooperate in folding of proteins.

Folding in the endoplasmic reticulum The eukaryotic cell has a specialized compartment, ER, where folding occurs for the proteins that are destined for secretion to an extracellular environment or to the plasma membrane and other organelles in the secretory pathway. The ER lumen is more likely to the extracellular space than the cytosol with respect to high calcium concentration and high oxidizing potential. It contains various types of chaperones and folding factors not only to assist efficient folding of proteins but also to play a qualitycontrol role (Sitia and Braakman, 2003; Welch, 2004). A variety of quality control mechanisms operate in the ER and in downstream compartments of the secretory pathway to ensure the fidelity and regulation of protein expression. Only proteins that fold and mature properly pass a stringent selection process and go their own ways to target compartments. If proper maturation fails, a complex degradation machinery eliminates misfolded or unassembled secretory proteins from the ER (Plemper and Wolf, 1999). The proteins are retained in 
Table 1. Some human conformational diseases caused by protein deposits

\begin{tabular}{|c|c|c|}
\hline Disease & Disease proteins & Characteristic pathology \\
\hline $\begin{array}{l}\text { Familial encephalopathy with } \\
\text { neuroserpin inclusion bodies }\end{array}$ & Neuroserpin & Collins body formation; neuronal inclusions \\
\hline$\alpha_{1}$-antitrypsin deficiency & $\alpha_{1}$-antitrypsin & $\begin{array}{l}\text { Inclusions in hepatocytes leading to emphysema, } \\
\text { liver cirrhosis }\end{array}$ \\
\hline Alzheimer's disease & $\begin{array}{l}\text { Amyloid } \beta \text {-protein and } \\
\text { hyperphosphorylated tau }\end{array}$ & Extracellular plaques; Tangles in neuronal cytoplasm \\
\hline Parkinson's disease & $\alpha$-synuclein & Lowy body formation \\
\hline Creutzfeldt-Jakob disease & Prion protein $\left(\mathrm{PrP}^{\mathrm{sc}}\right)$ & $\begin{array}{l}\text { Spongiform degeneration; extracellular plaques; } \\
\text { amyloid inside and outside neurons }\end{array}$ \\
\hline $\begin{array}{l}\text { Huntington's disease and other } \\
\text { polyglutamine expansion disease }\end{array}$ & $\begin{array}{l}\text { Long glutamine stretches within } \\
\text { certain proteins }\end{array}$ & Intranuclear inclusions and cytoplasmic aggregates \\
\hline
\end{tabular}

Adapted from the references (Lomas and Carrell, 2002; Selkoe, 2003).

an ER/pre-Golgi compartment and then hydrolyzed by the cytosolic ubiquitin-proteasome system. This requires retrograde translocation of proteins from the ER back to the cytoplasm, which is mediated by Sec61, the central component of the ER protein-import channel (Pilon et al., 1997).

The ER quality control pathway operates at several levels and by multiple mechanisms. All newly synthesized proteins entering the ER are subject to a primary quality control that monitors their architectural design through ubiquitous folding sensors. Chaperones of the Hsp70 (BiP) and Hsp90 (GRP94) families recognize redundant structural features of unfolded polypeptides and assist in their folding and assembly. BiP and GRP94, with other folding factors, seem to form an ER network that can bind to unfolded protein substrates instead of existing as free pools that assemble onto substrate proteins (Meunier et al., 2002). The oxidoreductases of the protein disulfide isomerase (PDI) family are required for correct formation of disulfide bonds (Freedman et al., 1989). The PDIs are abundant in secretory cells and catalyses thiol:protein-disulfide interchange with very broad protein substrate specificity. Calnexin and calreticulin add or remove carbohydrate moieties. These lectin chaperones and their associated co-chaperone ERp57, a glycoprotein specific thioldisulfide oxidoreductase, act as sensors to monitor and promote efficient folding of a wide range of glycoproteins (Ellgaard and Frickel, 2003). Secondary quality control mechanisms rely on cell-specific factors and facilitate export of individual proteins. Like the primary quality control factors, secondary factors promote the folding, maturation and assembly of their substrates. They are often present only in cell types which produce the respective substrate proteins. Examples include Hsp47, which transiently associates with procollagen and is involved in collagen processing and prevention of the secretion of procollagen with abnormal conformation (Nagata, 1996), and the receptor-associated protein (RAP), which exerts chaperone action on certain LDL-receptor family members (Bu and Schwartz, 1998).

Cellular machinery regulating synthesis, translocation, folding and degradation of proteins seems to operate in a very stringent manner to ensure protein aggregation is minimized. Nevertheless, in a certain disease condition, misfolded protein will escape such elaborate system forming protein aggregates. In the next section, we will pay attention to protein misfolding and the relevant human diseases

\section{Protein Misfolding and Conformational Diseases}

Conformational diseases and the protein deposits Protein misfolding gives rise to the malfunctioning of living systems. Diverse diseases have been shown to arise from protein misfolding and can be grouped as conformational diseases (Table 1). Typical examples are serpinopathies such as $\alpha_{1}$ antitrypsin deficiency leading to emphysema or liver cirrhosis and familial encephalopathy with neuroserpin inclusion bodies, and various neurodegenerative disorders such as Alzheimer's disease, Huntington's disease, Parkinson's disease and the prion diseases (Lomas and Carrell, 2002; BossyWetzel et al., 2004; Stefani, 2004). In these disorders, specific peptides or proteins misfold, often as a result of mutations, and give rise to protein aggregates. In serpinopathies, serpin molecules such as $\alpha_{1}$-antitrypsin and neuroserpin form loopsheet polymers. In Alzheimer's disease, extracellular amyloid$\beta$ peptide deposition is thought to be intimately associated with the disease. Several genetic loci related to Parkinson's disease were found and one of them is $\alpha$-synuclein which forms intracellular aggregates. Huntington's disease is caused by a mutant version of the protein huntingtin. It has a longer expansion of amino-terminal polyglutamine domain and thus is more prone to aggregation. Prion diseases, such as Creutzfeldt-Jakob disease, are caused by deposition of prion protein aggregates in the brain and nervous system (Prusiner et al., 1998; Selkoe, 2003).

Some of the diseases result from loss-of-function. In the case of $\alpha_{1}$-antitrypsin deficiency, misfolded $\alpha_{1}$-antitrypsin is retained in hepatocyte and secretion to blood plasma is blocked (Yu et al., 1995). The lack of circulating $\alpha_{1}$ antitrypsin induces an imbalance between the anti-proteolytic 
activity and elastase, which causes onset of emphysema due to failure to protect elastic tissue of the lung from proteolysis by elastase (Lomas and Carrell, 2002). In other cases like the neurodegenerative diseases, misfolded proteins escape the protective mechanisms and form intractable aggregates within cells or in the extracellular space. It is thought that either the aggregates of the disease protein themselves or the work done by the aggregates, or the process of their formation confers cellular toxicity. This idea supports the notion that misfolded disease proteins act through a gain-of-function (Selkoe, 2003).

Properties of amyloid fibril and the mechanism of its formation The disease proteins involved in conformational disorders have no obvious sequence similarities. Moreover, the molecular mechanisms involved are diverse and distinct with each disease. However, the aggregates, into which they can convert, share a common structural feature. Almost all aggregations result in $\beta$-linkages formed by hydrogen bonding between peptide loops and sheets. In $\alpha_{1}$-antitrypsin deficiency, some point mutations destabilize central $\beta$-sheet to allow incorporation of a loop of another molecule into the $\beta$-sheet. Sequential loop-insertion results in chains of polymers (Lomas et al., 1992). Domain swapping is another mechanism of intermolecular linkage in cystatin polymer (Staniforth et al., 2001). In these polymers, the individual molecules substantially retain their ordered structure. However, in other proteins, such as $\beta_{2}$-microglobulin (McParland et al., 2002) and the proteins involved in neurodegenerative diseases, linkage is formed by the remarkable realignment of peptide segments to give the sequential layering of $\beta$-structures known as $\beta$-amyloid fibril. No structure of an amyloid fibril has yet been determined in atomic detail. However, results from the experiments by X-ray fibre diffraction, cryoelectron microscopy and solid-state NMR have shown that amyloid fibrils are long, unbranched and often twisted structures a few nanometres in diameter and the organized core structure is composed of $\beta$-sheets whose strands run perpendicular to the fibril axis (Sunde and Blake, 1997; Jimenez et al., 1999; Petkova et al., 2002).

Under appropriate circumstances, misfolded monomers may oligomerize into pre-fibrillar assemblies. Such circumstances can be satisfied by extreme conditions of $\mathrm{pH}$ and temperature or partial proteolysis for non-disease proteins, whereas destabilizing mutations on disease proteins can induce such transition even under physiological conditions. The intrinsic effect of specific mutations on the rate of aggregation can be correlated to a remarkable extent with the changes in simple physicochemical properties such as hydrophobicity, secondary structure propensity and charge (Chiti et al., 2003). Generally, aggregation occurs in two steps. The first step characterized by a slow lag phase seems to involve the formation of soluble oligomers as a result of relatively nonspecific interactions. The oligomeric nucleus then rapidly grows. The lag phase can be minimized or eliminated by seeding preformed nucleus (Caughey and Lansbury, 2003 Soto, 2003 \#58).
The accumulation of toxic misfolded proteins may overload the quality control system. Unfolding of substrate proteins is the prerequisite for degradation by the proteasome, and $\beta$ structure is more difficult to unravel than $\alpha$-helix or surface loop. This explains why amyloid deposits, having high content of $\beta$-structure, are not easily cleared away by the proteasome system (Lee et al., 2001). The undegradable deposits may in turn sequestrate components of the chaperone and degradation systems, reducing the activity of assisted folding and proteolysis. The whole process will form a positive feed back cycle leading to cellular toxicity. Aberrant interactions of misfolded proteins with other proteins will also cause cellular toxicity because the interacting proteins are then no more functional. For example, polyglutamine-expanded huntingtin can recruit other important cellular proteins with normal polyglutamine stretches into the aggregates (Sakahira et al., 2002). Another model on the pathophysiology of amyloid protein has emerged. According to the channel hypothesis, aggregated protein forms a ring-like structure and inserts into the cellular membrane as a pore, which induces cytotoxicity including calcium dysregulation, membrane depolarization, mitochondrial dysfunction, and inhibition of long-term potentiation (Kagan et al., 2004). It is important to note that those mechanisms described above are not mutually exclusive but complementary to each other and may act in combination.

\section{Concluding Remark}

Here we briefly review general mechanism of protein folding in the cells and conformational diseases caused by protein misfolding and aggregation. Human diseases characterized by insoluble deposits of proteins have been recognized for almost two centuries. Many efforts have been devoted to reveal the detailed mechanism of protein folding and the molecular pathway to conformational diseases. However, the precise mechanism in which natively soluble proteins undergo partial unfolding and aberrant refolding to produce amyloid fibrils, is still unclear. Understanding of how protein folding is regulated and characterization of amyloid deposits found in the conformational diseases will help us to get a vision on the molecular mechanism of amyloid formation. In this respect, it is noteworthy that protein unfolding is also regulated inside of cells (Matouschek, 2003). Conformational transition of proteins in the cell including folding, unfolding, misfolding, and aggregation remains to be the center of biologists' interests.

Acknowledgments The authors are grateful for the grant from Functional Proteomics Research Center of the $21^{\text {st }}$ Century Frontier Research Program funded by the Korean Ministry of Science and Technology. 


\section{References}

Baram, D. and Yonath, A. (2005) From peptide-bond formation to cotranslational folding: dynamic, regulatory and evolutionary aspects. FEBS Lett. 579, 948-954.

Barouch, W., Prasad, K., Greene, L. and Eisenberg, E. (1997) Auxilin-induced interaction of the molecular chaperone Hsc70 with clathrin baskets. Biochemistry 36, 4303-4308.

Barral, J. M., Broadley, S. A., Schaffar, G. and Hartl, F. U. (2004) Roles of molecular chaperones in protein misfolding diseases. Semin. Cell Dev. Biol. 15, 17-29.

Bossy-Wetzel, E., Schwarzenbacher, R. and Lipton, S. A. (2004) Molecular pathways to neurodegeneration. Nat. Med. 10 (suppl), S2-S9.

Braig, K., Otwinowski, Z., Hegde, R., Boisvert, D. C., Joachimiak, A., Horwich, A. L. and Sigler, P. B. (1994) The crystal structure of the bacterial chaperonin GroEL at $2.8 \AA$. Nature 371, 578-586.

$\mathrm{Bu}$, G. and Schwartz, A. L. (1998) RAP, a novel type of ER chaperone. Trends Cell Biol. 8, 272-276.

Bukau, B. and Horwich, A. L. (1998) The Hsp70 and Hsp60 chaperone machines. Cell 92, 351-366.

Caughey, B. and Lansbury, P. T. (2003) Protofibrils, pores, fibrils, and neurodegeneration: separating the responsible protein aggregates from the innocent bystanders. Annu. Rev. Neurosci. 26, 267-298.

Chiti, F., Stefani, M., Taddei, N., Ramponi, G. and Dobson, C. M. (2003) Rationalization of the effects of mutations on peptide and protein aggregation rates. Nature 424, 805-808.

Dill, K. A. and Chan, H. S. (1997) From Levinthal to pathways to funnels. Nat. Struct. Biol. 4, 10-19.

Dobson, C. M. (2003) Protein folding and misfolding. Nature 426, 884-890.

Dobson, C. M. (2004) Principles of protein folding, misfolding and aggregation. Semin. Cell Dev. Biol. 15, 3-16.

Ellgaard, L. and Frickel, E. M. (2003) Calnexin, calreticulin, and ERp57: teammates in glycoprotein folding. Cell Biochem. Biophys. 39, 223-247.

Ellis, R. J. (2001a) Macromolecular crowding: an important but neglected aspect of the intracellular environment. Curr. Opin. Struct. Biol. 11, 114-119.

Ellis, R. J. (2001b) Molecular chaperones: inside and outside the Anfinsen cage. Curr. Biol. 11, R1038-1040.

Fersht, A. R. (1999) Folding pathways and energy landscapes; in Structure and mechanism in protein science: A guide to enzyme catalysis and protein folding, Julet, M. R. and Hadler, G. L. (eds.), pp. 573-614, W H Freeman and Co., New York, USA.

Fersht, A. R. and Daggett, V. (2002) Protein folding and unfolding at atomic resolution. Cell 108, 573-582.

Flaherty, K. M., McKay, D. B., Kabsch, W. and Holmes, K. C. (1991) Similarity of the three-dimensional structures of actin and the ATPase fragment of a $70-\mathrm{kDa}$ heat shock cognate protein. Proc. Natl. Acad. Sci. USA 88, 5041-5045.

Freedman, R. B., Bulleid, N. J., Hawkins, H. C. and Paver, J. L. (1989) Role of protein disulphide-isomerase in the expression of native proteins. Biochem. Soc. Symp. 55, 167-192.

Frydman, J. and Hartl, F. U. (1996) Principles of chaperoneassisted protein folding: differences between in vitro and in vivo mechanisms. Science 272, 1497-1502.

Grantcharova, V., Alm, E. J., Baker, D. and Horwich, A. L.
(2001) Mechanisms of protein folding. Curr. Opin. Struct. Biol. 11, 70-82.

Hardesty, B. and Kramer, G. (2001) Folding of a nascent peptide on the ribosome. Prog. Nucleic Acid Res. Mol. Biol. 66, 41-66.

Hartl, F. U. and Hayer-Hartl, M. (2002) Molecular chaperones in the cytosol: from nascent chain to folded protein. Science $\mathbf{2 9 5}$, 1852-1858.

Jimenez, J. L., Guijarro, J. I., Orlova, E., Zurdo, J., Dobson, C. M., Sunde, M. and Saibil, H. R. (1999) Cryo-electron microscopy structure of an $\mathrm{SH} 3$ amyloid fibril and model of the molecular packing. EMBO J. 18, 815-821.

Kagan, B. L., Azimov, R. and Azimova, R. (2004) Amyloid peptide channels. J. Membr. Biol. 202, 1-10.

Karzai, A. W. and McMacken, R. (1996) A bipartite signaling mechanism involved in DnaJ-mediated activation of the Escherichia coli DnaK protein. J. Biol. Chem. 271, 1123611246.

Laufen, T., Mayer, M. P., Beisel, C., Klostermeier, D., Mogk, A., Reinstein, J. and Bukau, B. (1999) Mechanism of regulation of hsp70 chaperones by DnaJ cochaperones. Proc. Natl. Acad. Sci. USA 96, 5452-5457.

Lee, C., Schwartz, M. P., Prakash, S., Iwakura, M. and Matouschek, A. (2001) ATP-dependent proteases degrade their substrates by processively unraveling them from the degradation signal. Mol. Cell 7, 627-637.

Lomas, D. A. and Carrell, R. W. (2002) Serpinopathies and the conformational dementias. Nat. Rev. Genet. 3, 759-768.

Lomas, D. A., Evans, D. L., Finch, J. T. and Carrell, R. W. (1992) The mechanism of $\mathrm{Z} \mathrm{a}_{1}$-antitrypsin accumulation in the liver. Nature 357, 605-607.

Matouschek, A. (2003) Protein unfolding--an important process in vivo? Curr. Opin. Struct. Biol. 13, 98-109.

Mayer, M. P. and Bukau, B. (2005) Hsp70 chaperones: cellular functions and molecular mechanism. Cell. Mol. Life Sci. 62, 670-684.

McParland, V. J., Kalverda, A. P., Homans, S. W. and Radford, S. E. (2002) Structural properties of an amyloid precursor of $\beta_{2}-$ microglobulin. Nat. Struct. Biol. 9, 326-331.

Meunier, L., Usherwood, Y. K., Chung, K. T. and Hendershot, L. M. (2002) A subset of chaperones and folding enzymes form multiprotein complexes in endoplasmic reticulum to bind nascent proteins. Mol. Biol. Cell 13, 4456-4469.

Munro, S. and Pelham, H. R. (1986) An Hsp70-like protein in the ER: identity with the $78 \mathrm{kd}$ glucose-regulated protein and immunoglobulin heavy chain binding protein. Cell 46, 291-300.

Nagata, K. (1996) Hsp47: a collagen-specific molecular chaperone. Trends Biochem. Sci. 21, 22-26.

Petkova, A. T., Ishii, Y., Balbach, J. J., Antzutkin, O. N., Leapman, R. D., Delaglio, F. and Tycko, R. (2002) A structural model for Alzheimer's $\beta$-amyloid fibrils based on experimental constraints from solid state NMR. Proc. Natl. Acad. Sci. USA 99, 16742-16747.

Pilon, M., Schekman, R. and Romisch, K. (1997) Sec61p mediates export of a misfolded secretory protein from the endoplasmic reticulum to the cytosol for degradation. EMBO J. 16, 4540-4548.

Plemper, R. K. and Wolf, D. H. (1999) Retrograde protein translocation: ERADication of secretory proteins in health and disease. Trends Biochem. Sci. 24, 266-270.

Prusiner, S. B., Scott, M. R., DeArmond, S. J. and Cohen, F. E. 
(1998) Prion protein biology. Cell 93, 337-348.

Rudiger, S., Germeroth, L., Schneider-Mergener, J. and Bukau, B. (1997) Substrate specificity of the DnaK chaperone determined by screening cellulose-bound peptide libraries. EMBO J. 16, 1501-1507.

Sakahira, H., Breuer, P., Hayer-Hartl, M. K. and Hartl, F. U. (2002) Molecular chaperones as modulators of polyglutamine protein aggregation and toxicity. Proc. Natl. Acad. Sci. USA 99, 16412-16418.

Selkoe, D. J. (2003) Folding proteins in fatal ways. Nature 426, 900-904.

Siegers, K., Bolter, B., Schwarz, J. P., Bottcher, U. M., Guha, S. and Hartl, F. U. (2003) TRiC/CCT cooperates with different upstream chaperones in the folding of distinct protein classes. EMBO J. 22, 5230-5240.

Sitia, R. and Braakman, I. (2003) Quality control in the endoplasmic reticulum protein factory. Nature 426, 891-894.

Staniforth, R. A., Giannini, S., Higgins, L. D., Conroy, M. J., Hounslow, A. M., Jerala, R., Craven, C. J. and Waltho, J. P. (2001) Three-dimensional domain swapping in the folded and molten-globule states of cystatins, an amyloid-forming structural superfamily. EMBO J. 20, 4774-4781.

Stefani, M. (2004) Protein misfolding and aggregation: new examples in medicine and biology of the dark side of the protein world. Biochim. Biophys. Acta 1739, 5-25.

Sunde, M. and Blake, C. (1997) The structure of amyloid fibrils by electron microscopy and X-ray diffraction. Adv. Protein Chem. 50, 123-159.

Thulasiraman, V., Yang, C. F. and Frydman, J. (1999) In vivo newly translated polypeptides are sequestered in a protected folding environment. $E M B O \mathrm{~J} . \mathbf{1 8}, 85-95$.

Welch, W. J. (2004) Role of quality control pathways in human diseases involving protein misfolding. Semin. Cell Dev. Biol. 15, 31-38.

Yu, M. H., Lee, K. N. and Kim, J. (1995) The Z type variation of human $\mathrm{a}_{1}$-antitrypsin causes a protein folding defect. Nat. Struct. Biol. 2, 363-367.

Zhu, X., Zhao, X., Burkholder, W. F., Gragerov, A., Ogata, C. M., Gottesman, M. E. and Hendrickson, W. A. (1996) Structural analysis of substrate binding by the molecular chaperone DnaK. Science 272, 1606-1614. 